\title{
Amplitude Response of Single-Wall Carbon Nanotube Probes during Tapping Mode Atomic Force Microscopy: Modeling and Experiment
}

2006

Vol. 6, No. 8

$1669-1673$

\author{
A. Kutana, K. P. Giapis, ${ }^{\star}$ J. Y. Chen, and C. P. Collier \\ Division of Chemistry and Chemical Engineering, California Institute of Technology, \\ Pasadena, California 91125
}

Received April 13, 2006; Revised Manuscript Received July 6, 2006

\begin{abstract}
Imaging of surfaces with carbon nanotube probes in tapping mode results frequently in complex behavior in the amplitude-distance curves monitored. Using molecular mechanics simulations, we calculate the force exerted on a nanotube pressed against a smooth surface as it undergoes deformation and buckling. This nonlinear force is then used in a macroscopic equation, describing the response of a damped harmonic oscillator, to predict the amplitude response of a nanotube AFM probe. Similarities between the prediction and experiment suggest that the complex amplitude response seen in the experiment may be explained by the nonlinearity in the force exerted on the nanotube and thus must not necessarily be related to the structure of the surface.
\end{abstract}

Single-wall carbon nanotubes (SWNTs) used as atomic force microscopy (AFM) probes have several advantages over conventional silicon tips due to their strength, elasticity, high aspect ratio and smaller dimensions. ${ }^{1}$ For example, they are chemically inert and can sustain significant loads without breaking, although they routinely achieve better resolution than conventional tips. In addition, they are capable of nondestructive imaging of soft samples such as organic molecules. ${ }^{2}$ However, when used in tapping mode imaging, the carbon nanotube attached to the AFM tip may deform, thereby influencing the amplitude response recorded and complicating its interpretation.

Although deformations in carbon nanotubes with both ends fixed are well understood, ${ }^{3}$ deformations in nanotubes with one end free, which are important in imaging applications of nanotube AFM probes, have been studied less. Modeling of such interactions was done with simple macroscopic models, ${ }^{4,5}$ or by considering only linear elastic deformations. ${ }^{6-8}$ A better understanding of the response dynamics of nanotube AFM probes could help to better assess their capabilities and limitations in imaging. In this Letter, we present a full atomistic description of a nanotube AFM probe forced into contact with a surface using molecular mechanics simulations based on a realistic interatomic potential for carbon. The simulations yield a force response describing the nanotube nonlinear elastic deformation dynamics. The force response is then used to predict the macroscopic amplitude of the AFM

\footnotetext{
* Corresponding author. E-mail: giapis@cheme.caltech.edu.
}

cantilever in tapping mode imaging, which is compared to experimental results.

The fabrication and characterization of SWNT tips used in the AFM experiment has been described previously. ${ }^{9}$ Briefly, SWNTs were grown by chemical vapor deposition on a silicon wafer decorated with iron nanoparticles. These SWNTs were attached to gold-coated silicon AFM tips (Multi-75, Budget Sensors) to form "nanotube probes" using the pickup technique of Hafner et al. ${ }^{10}$ A Digital Instruments (Santa Barbara, CA) Multimode AFM with a Nanoscope IV controller was used for this work. Subsequently, forcedistance curves of these nanotube probes were generated, which consist of scans of the damped oscillation amplitude as a function of the average tip-sample separation for a given cantilever driving voltage.

Experimental observations of imaging with nanotube AFM probes in the tapping mode suggested that the protruding nanotube probe might buckle at some point after establishing contact with the surface. The experimental oscillation amplitude of the probe displays a complex response as a function of the equilibrium tip-surface distance $(Z)$. An example of the complex behavior is shown in Figure 1 for a $5 \pm 1 \mathrm{~nm}$ diameter, $100 \mathrm{~nm}$ long SWNT tip tapping on a highly-ordered pyrolytic graphite (HOPG) surface. A pattern of maxima and minima at different distances to the surface is observed for free-air amplitudes of 14, 26, and $42 \mathrm{~nm}$. A question arises as to whether these patterns appear as a result of multiple probe buckling or surface irregularities or 


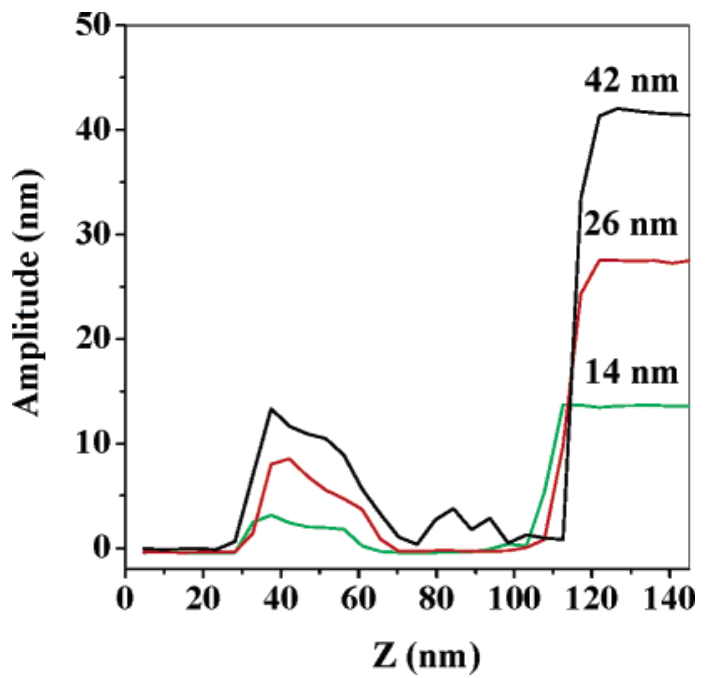

Figure 1. Experimental amplitude-distance curves from AFM imaging of an HOPG surface in tapping mode. A single-walled carbon nanotube of length $L=100 \mathrm{~nm}$ and diameter $5 \pm 1 \mathrm{~nm}$ was used. Curves for free air oscillation amplitudes of 14, 26, and $42 \mathrm{~nm}$ are shown.

displaced extraneous matter on the surface. It is shown herein that the nonlinearity in the force response resulting from a single buckling of a nanotube on a smooth surface can give rise to such complex behavior.

The molecular mechanics (MM) simulations of nanotube deformations during AFM imaging were performed with a code developed by Brenner, ${ }^{11}$ which implements a reactive bond order (REBO) potential for hydrocarbons. ${ }^{11}$ REBO was shown to correctly reproduce the energy of a variety of structural arrangements of carbon, including a 3-coordinated configuration in a graphene sheet. The potential has shown a great degree of transferability and should be capable of describing changes in local geometry such as those occurring during both linear and nonlinear elastic deformations in nanotube walls. Previously, REBO has been extensively used for modeling of carbon-based nanostructures and applied to simulations of mechanical deformations in nanotubes. ${ }^{12-15}$

The main configuration representing the interaction of the nanotube probe with a flat substrate used in current simulations is shown in Figure 2. The embedded top end of the nanotube is initially slanted, mimicking its attachment parallel to the side of the pyramidal silicon tip, for which typical half cone angles are in the range $20-30^{\circ}$. Because only nearest neighbor interactions between carbon atoms are considered, it was sufficient to immobilize atoms within 10 $\AA$ of the top of the nanotube, whereas the rest of the atoms were allowed to move according to forces acting on them from other atoms and the substrate.

The HOPG substrate was modeled with a continuous wall potential ${ }^{16}$ representing an average force field created by atoms occupying a bottom half space with number density $n$. The interaction of each atom in the nanotube with the HOPG surface is given by the wall potential $U(z)$ :

$$
U(z)=n \int_{z^{\prime}>z} V\left(r^{\prime}\right) \mathrm{d}^{3} r^{\prime}
$$

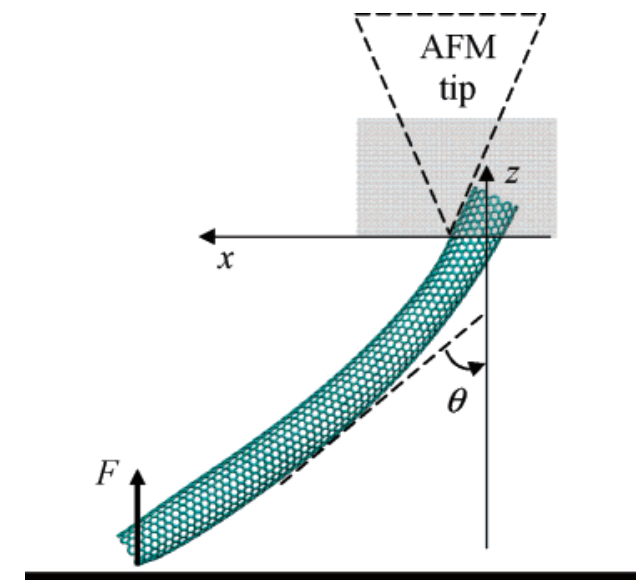

Figure 2. Carbon nanotube geometry considered for the MM simulations. The force $F$ exerted by the surface on the nanotube depends on its vertical position $z$. At each point of the nanotube, a local bending angle $\theta$ is defined as shown.

where $V(r)$ is the carbon-carbon pair interaction (van der Waals type), $z$ is the distance from the atom to the surface, and $n=0.114 \AA^{-3}$ is the number density of carbon atoms in the graphite substrate. Assuming a Lennard-Jones (LJ) form for the pairwise interaction, the wall potential becomes

$$
U(z)=4 \epsilon \pi n \sigma^{3}\left(\frac{1}{45}\left(\frac{\sigma}{z}\right)^{9}-\frac{1}{6}\left(\frac{\sigma}{z}\right)^{3}\right)
$$

where $\sigma=3.407 \AA$ and $\epsilon / k_{\mathrm{B}}=34.4 \mathrm{~K}$ are the carboncarbon LJ length and energy parameters, obtained by matching the elastic constant $c_{33}$ and equilibrium interplanar distance for graphite, in agreement with ref 17 . This idealized model omits frictional forces in the plane of the substrate, which may result from atomic corrugations on the surface.

The force-distance curves were obtained by externally moving the top of the tube along the normal to the surface in small steps of $0.1 \AA$ and minimizing the energy of the system for each step using the conjugate gradient method. Any thermal atomic motions were omitted from the model to avoid thermal noise in the force response.

Because tubes with experimental sizes (100 nm length, 5 $\mathrm{nm}$ diameter) could not be simulated due to computational power limits, smaller nanotubes (13.7 nm length, 1.3-2.0 $\mathrm{nm}$ diameter) were considered instead. Figure 3 shows snapshots of a geometrical shape of a $(10,10)$ nanotube with the length of the free end $L=13.7 \mathrm{~nm}$ (14.8 nm total length) and initial tilt of $30^{\circ}$ with respect to the surface normal pushed against a flat substrate. In Figure 3a, the free end of the suspended nanotube does not contact the surface. The elastically deformed nanotube just before buckling is shown in Figure $3 b$, while the configuration immediately after buckling is captured in Figure 3c. The final state of a fully lowered tube is shown in Figure 3d. The force response for this nanotube as a function of the probe-surface distance at three different tilt angles of $20^{\circ}, 25^{\circ}$, and $30^{\circ}$ is shown in Figure 4, with the four arrows indicating the position of a $30^{\circ}$ tilted nanotube in each of the snapshots of Figure 3.

On the initial approach to the surface, there is a negative force due to a van der Waals attraction between the tube 


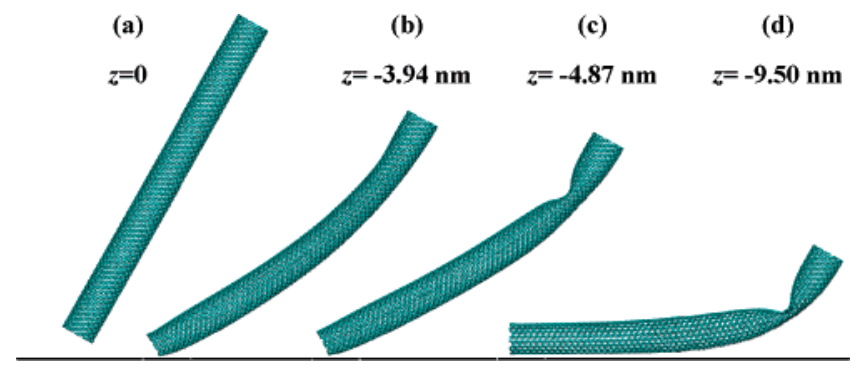

Figure 3. Snapshots of a geometrical shape of a $(10,10)$ nanotube with a length $L=13.7 \mathrm{~nm}$ and initial tilt of $30^{\circ}$ off-normal pushed against a flat substrate at different distances from the surface. (a) The free end of the suspended nanotube does not contact the surface. (b) The nanotube is elastically deformed just before buckling. (c) The nanotube is nonlinearly deformed after buckling. (d) The final state of the fully lowered nanotube is shown.

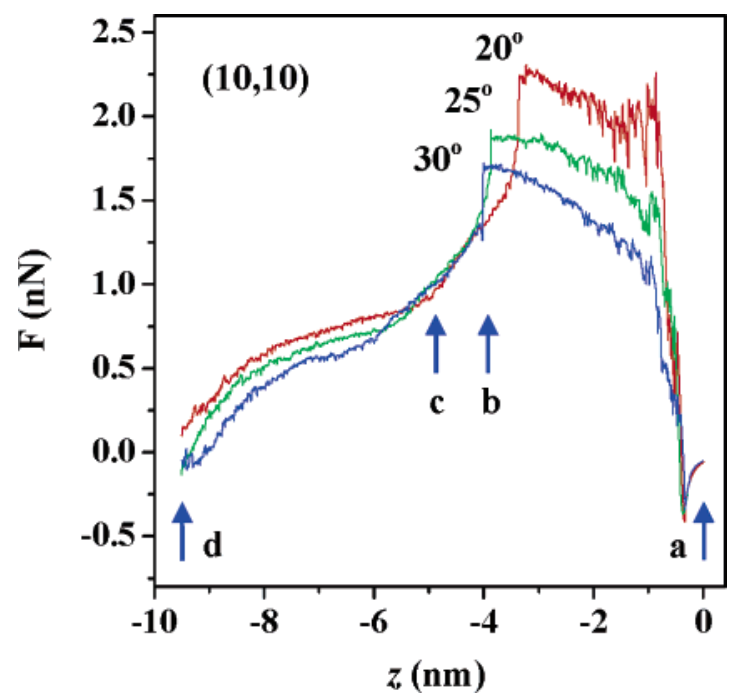

Figure 4. Calculated force responses for a $(10,10)$ nanotube probe with a length $L=13.7 \mathrm{~nm}$ pushed against a flat HOPG surface as a function of the probe-surface distance at three different tilt angles of $20^{\circ}, 25^{\circ}$, and $30^{\circ}$. The four arrows indicate the position of a $30^{\circ}$ inclined nanotube corresponding to each of the snapshots of Figure 3.

and the surface, which causes a small dip in the forcedistance curve, followed by a sign reversal when the force becomes repulsive. The repulsive interaction results in a rapidly growing force on the nanotube, defining a region of elastic behavior, which abruptly terminates when buckling occurs. At that point, the magnitude of the force drops sharply. A further decrease in the probe-surface distance brings about a more gradual decrease in force. As expected, the initial stiffness of the nanotube increases as the probe tilt angle changes from $30^{\circ}$ to $20^{\circ}$, resulting in buckling at a smaller absolute $z$ value.

The responses for nanotubes of varying diameters with a constant length of $13.7 \mathrm{~nm}$ are shown in Figure 5. The curves are generally similar for $(10,10),(12,12)$, and $(15,15)$ nanotubes, showing a trend of increasing probe stiffness and earlier buckling with increasing tube diameter. A remarkable feature of all these curves, including those for different tilts and diameters, is that they nearly collapse on each other after the buckling point, underlining the similarity of the bending $30^{\circ}$ tilt

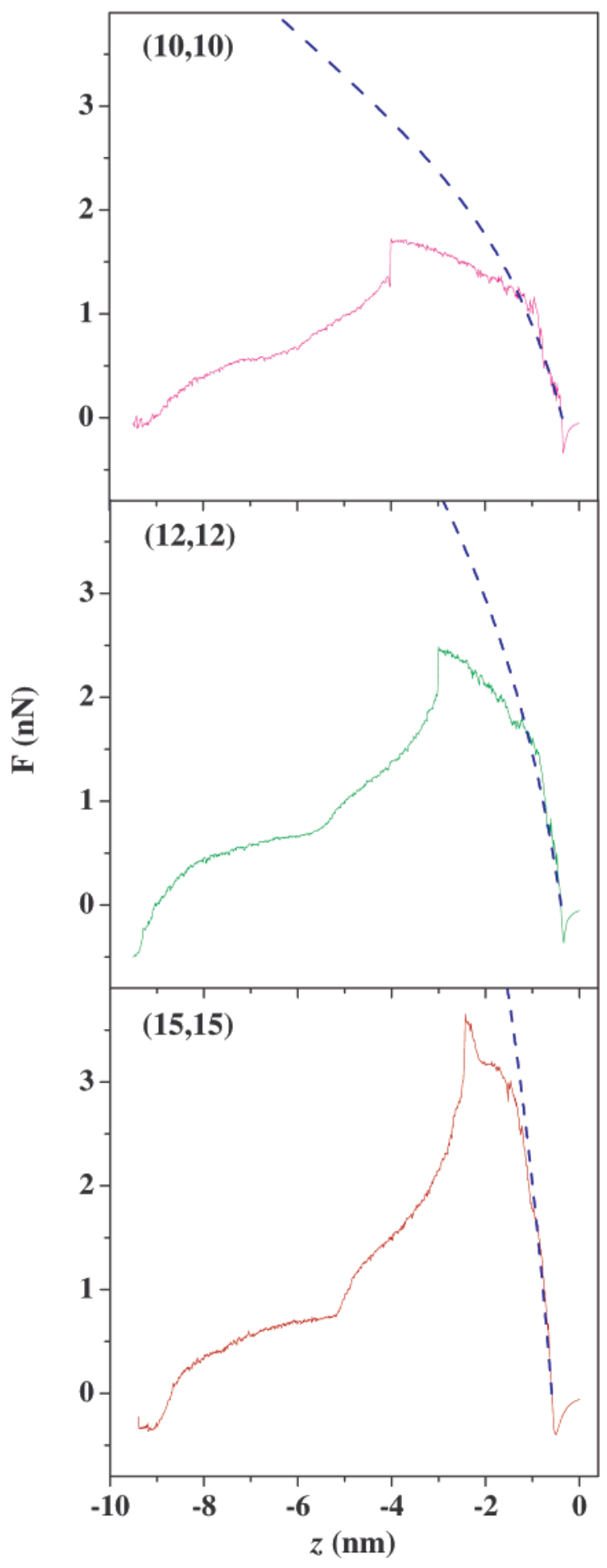

Figure 5. Calculated force responses for tubes of varying diameters and constant length of $13.6 \mathrm{~nm}$ and tilt of $30^{\circ}$ pushed against a flat HOPG surface for (a) $(10,10)$, (b) $(12,12)$, and (c) $(15,15)$ nanotubes. Solid curves are obtained from MM simulations, dashed curves are solutions of eq 3 for elastic bending of a cantilever rod.

force in the deformed tubes. Unlike macroscopic tubes, the buckling deformation in nanotubes is not permanent, and the original shape is completely restored after the load has been removed. ${ }^{18,19}$ This behavior has been verified in simulations (not shown here) by lifting the tube from the surface. 
The initial elastic deformation in nanotubes may be considered from the point of view of the continuum theory of elasticity, by representing the tube as a continuous elastic rod with a circular cross section. The elastic bending of a cantilever rod with one embedded end and a force $F$ applied to the other end (Figure 2) is described by ${ }^{20}$

$$
\mathrm{IE} \frac{\mathrm{d}^{2} \theta}{\mathrm{d} l^{2}}-F \sin \theta=0
$$

where $l$ is the distance along the rod measured from the top, $\theta(l)$ is the bending angle, $E$ is the Young's modulus of the rod material, and $I=\pi\left(R_{2}{ }^{4}-R_{1}{ }^{4}\right) / 4$ for a rod with a circular cross section whose inner and outer radii are $R_{1}$ and $R_{2}$. Equation 3 can be solved numerically ${ }^{21}$ for a rod of length $L$ using the boundary condition $\theta(0)=\alpha$, where $\alpha$ is the tilt angle at the upper end, and $\mathrm{d} \theta /\left.\mathrm{d} l\right|_{l=L}=0$ at the lower end. Young's modulus $E$ was determined from MM simulations of uniform axial compression of infinite nanotubes, yielding the values of 1055,1052 , and $1049 \mathrm{GPa}$ for $(10,10)$, $(12,12)$, and $(15,15)$ nanotubes, respectively, assuming a tube wall thickness of $3.4 \AA$.

The resulting solution yields values for the force $F$ in the elastic approximation as a function of the change in the projection of the rod on the vertical axis. The results are superimposed as dashed lines on the force responses from the MM simulations of the $(10,10),(12,12)$, and $(15,15)$ nanotubes, as shown in Figure 5. The departure from the predictions of the beam model of the elasticity theory occurs earlier than the buckling point. Although no attempts to model the nonlinear elastic region have been made, the coincidence in behavior demonstrates that the elastic beam model offers a good approximation and that nanotubes indeed behave like macroscopic elastic bodies, at least at small deformations.

The calculated force curves may be used to predict the amplitude response of the interaction of a nanotube AFM probe with a surface to help interpret experimental observations. When imaging is done in tapping mode, the interaction of a nanotube tip with a surface is modeled ${ }^{22}$ as a damped harmonic oscillator according to

$$
\frac{\mathrm{d}^{2} z}{\mathrm{~d} t^{2}}=-\omega_{0}{ }^{2} z-\frac{\omega_{0}}{Q} \frac{\mathrm{d} z}{\mathrm{~d} t}+\frac{F(z)}{m}+\frac{f_{0}}{m} \cos \omega t
$$

Here, $z=z(Z, t)$ is the instantaneous SWNT tip-surface separation during the oscillation, which is equal to the equilibrium value $Z$ at zero driving force, $\omega_{0}$ is the resonant frequency of the oscillator, $Q$ is the quality factor, $m$ is the effective mass of the cantilever, $f_{0} \cos \omega t$ is the external force driving the oscillator at a frequency $\omega$, and $F(z)$ is the calculated response force acting on the probe from the surface. For the AFM system used in the current experiments, $\omega_{0} / 2 \pi=47.48 \mathrm{kHz}, m=5.39 \times 10^{-11} \mathrm{~kg}, Q=150$, and the driving force was operating at resonance frequency, $\omega$ $=\omega_{0}$. Using these values and the calculated $F(z)$, eq 4 was solved to model oscillations of a tip with a $(10,10)$ nanotube probe with length $L=13.6 \mathrm{~nm}$ and $30^{\circ}$ tilt angle. The free air oscillation amplitudes of 1.9, 3.5, and $5.7 \mathrm{~nm}$ were used

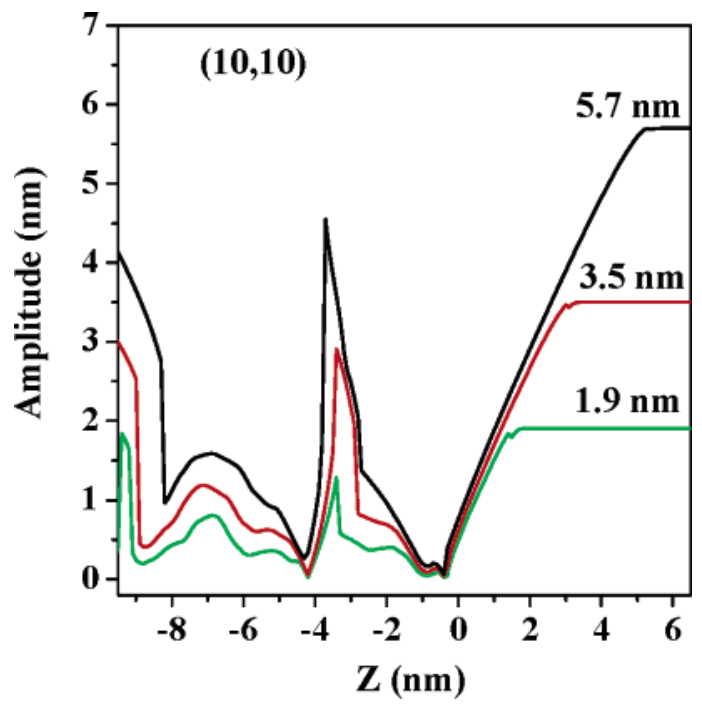

Figure 6. Calculated tapping mode AFM oscillation amplitudes of a $(10,10)$ nanotube probe as a function of the distance to the surface for three free-air amplitudes: $1.9,3.5$, and $5.7 \mathrm{~nm}$. The length of the probe is $13.6 \mathrm{~nm}$, and the tilt angle is $30^{\circ}$.

for a $13.6 \mathrm{~nm}$ long nanotube, obtained by scaling the experimental amplitudes for a $100 \mathrm{~nm}$ nanotube with the nanotube length. The calculated amplitudes are shown in Figure 6 and can be compared with the experimental results of Figure 1. As in the experiment, the calculated amplitude goes through minima and maxima as the distance to the surface changes. This behavior of the oscillator is not related to any features on the surface, which is ideally smooth in the simulations, but results from the nonlinear force on a nanotube undergoing buckling.

In conclusion, we have used molecular mechanics simulations to study the deformation dynamics of a carbon nanotube AFM probe used in imaging an HOPG surface in tapping mode. The initial deformation of the nanotube was found to be elastic and to be in agreement with the continuum theory of elasticity as applied to the bending of a cantilever rod. As the probe was pushed further toward the surface, the force on the nanotube increased, albeit deviating from the theory of elasticity, until buckling occurred, coinciding with an abrupt decrease in the force magnitude. As the nanotube was lowered further, the force continued to decrease, although gradually. The nonlinear force calculated was used as an input to a macroscopic equation describing the response of a damped harmonic oscillator, which was able to predict the amplitude response of a nanotube AFM probe used for imaging surfaces in tapping mode. Similarities between the simulation prediction and the experiment suggest that the complex amplitude response seen in the experiment may be explained by the nonlinearity in the force exerted on the nanotube and thus must not necessarily be related to the structure of the surface.

Acknowledgment. This work was supported in part by NSF (CTS-0508096).

\section{References}

(1) Hafner, J. H.; Cheung, C.-L.; Wooley, A. T.; Lieber, C. M. Prog. Biophys. Mol. Biol. 2001, 77, 73. 
(2) Bunch, J. S.; Rhodin, T. N.; McEuen, P. L. Nanotechnology 2004, $15, \mathrm{~S} 76$.

(3) Yakobson, B. I.; Brabec, C. J.; Bernholc, J. Phys. Rev. Lett. 1996, $76,2511$.

(4) Lee, S. I.; Howell, S. W.; Raman, A.; Reifenberger, R.; Nguyen, C. V.; Meyyappan, M. Nanotechnology 2004, 15, 416.

(5) Dubourg, F.; Aimé, J. P. Surf. Sci. 2000, 466, 137.

(6) Shapiro, I. R.; Solares, S. D.; Esplandiu, M. J.; Wade, L. A.; Goddard, W. A.; Collier, C. P. J. Phys. Chem. B 2004, 108, 13613.

(7) Solares, S. D.; Matsuda, Y.; Goddard, W. A., III. J. Phys. Chem. B 2005, 109, 16658.

(8) Solares, S. D.; Esplandiu, M. J.; Goddard, W. A.; Collier, C. P. J. Phys. Chem. B 2005, 109, 11493.

(9) Wade, L. A.; Shapiro, R. I.; Ma, Z.; Quake, S. R.; Collier, C. P. Nano Lett. 2004, 4, 725.

(10) Hafner, J. H.; Cheung, C.-L.; Oosterkamp, T. H.; Lieber, C. M. J. Phys. Chem. B 2001, 105, 743.

(11) Brenner, D. W.; Shenderova, O. A.; Harrison, J. A.; Stuart, S. J.; Ni, B.; Sinnott, S. B. J. Phys.: Solid State 2002, 14, 783.

(12) Guo, W.; Zhu, C. Z.; Yu, T. X.; Woo, C. H.; Zhang, B.; Dai, Y. T. Phys. Rev. Lett. 2004, 93, 245502.
(13) Liew, K. M.; Wong, C. H.; Tan, M. J. Appl. Phys. Lett. 2005, 87, 041901.

(14) Liew, K. M.; Hea, X. Q.; Wong, C. H. Acta Mater. 2004, 52, 2521.

(15) Elliott, J. A.; Sandler, J. K. W.; Windle, A. H.; Young, R. J.; Shaffer, M. S. P. Phys. Rev. Lett. 2004, 92, 095501.

(16) Steele, W. A. The Interaction of Gases with Solid Surfaces; Pergamon Press: Oxford, U.K., 1974.

(17) Lu, J. P.; Li, X.-P.; Martin, R. M. Phys. Rev. Lett. 1992, 68, 1551.

(18) Iijima, S.; Brabec, C.; Maiti, A.; Bernholc, J. J. Chem. Phys. 1996, 104, 2089.

(19) Falvo, M. R.; Clary, G. J.; Taylor, R. M.; Chi, V.; Brooks, F. P.; Washburn, S.; Superfine, R. Nature 1997, 389, 582.

(20) Landau, L. D.; Lifshitz, E. M. Theory of Elasticity; Pergamon Press: Oxford, U.K., 1986.

(21) Press, W. H.; Teukolsky, S. A.; Vetterling, W. T.; Flannery, B. P. Numerical Recipes in C; Cambridge University Press: Cambridge, U.K., 1992.

(22) García, R.; San Paulo, A. Phys. Rev. B 2000, 61, R13381.

NL0608310 